\title{
Imaging Defects in Quantum Materials
}

\author{
David C. Bell and Estelle Kalfon-Cohen
}

School of Engineering and Applied Sciences, Harvard University, Cambridge, MA 02138

The discovery of extraordinary new quantum materials with striking properties has caused great excitement, and promises to transform signal processing and computation. We have performed integrated research on three materials (1) Graphene [1-2] - electrons that move as massless particles at a constant speed; (2) Topological Insulators (TI) - mobile surface electrons with spins fixed to the direction of motion; and (3) Nitrogen-vacancy (NV) Centers in Diamond - a single spin stores a bit of quantum information (Fig. 1). Remarkably, the quantum phenomena displayed by these materials persists at room temperature, changing the rules for signal processing and computation and opening the way for quantum electronics.

Defects in materials effect the propagation of electrons and holes in graphene and topological insulators act in ways that are totally unlike carriers in conventional semiconductors - they move like twodimensional (2D) massless, ultra-relativistic electrons, except their speed is much less than the speed of light. Because there is no bandgap, an electron can pass through a potential barrier by temporarily turning into a hole, dramatically reducing scattering and improving coherence. In addition, for topological insulators the direction of the spin of a surface electron is tied to its direction of motion, providing an ideal means to transport spin information. In diamond, NV color center acts as like an isolated atom. More importantly the NV centers are by their very nature defects in the diamond structure and used to create diamond based quantum materials controlling the placement of these defects to produce the NV centers are is of critical importance. By exploiting the forbidden transitions in a NV center, one can coherently store a quantum bit of information in a spin at room temperature and sense very weak magnetic fields.

Aberration-corrected electron microscopy has been used to characterize MBE-grown films with high resolution at low beam voltages $(40 \& 80 \mathrm{kV})$ to directly visualize structural defects and relate them to performance. The imaging and analysis of defects in quantum materials presents new challenges on how to minimize surface and sample damage while imaging and analyzing structures at the direct atomic level (Fig. 2). The ability to directly image nitrogen vacancy centers directly in diamond would be a major break through which so far has proved elusive.

References:

[1] A. Reina, X. Jia, J. Ho, D. Nezich, H. Son, V. Bulovic, M.S. Dresselhaus, \& J. Kong, Nano Lett. 9, 30-35 (2008).

[2] K.S. Kim, Y. Zhao, H. Jang, S.Y. Lee, J.M. Kim, K.S. Kim, J.H. Ahn, P. Kim, J.Y. Choi, and B.H. Hong, Nature 457, 706-710 (2009).

[3] This work was supported by the STC Center for Integrated Quantum Materials, NSF Grant No. DMR-1231319. 


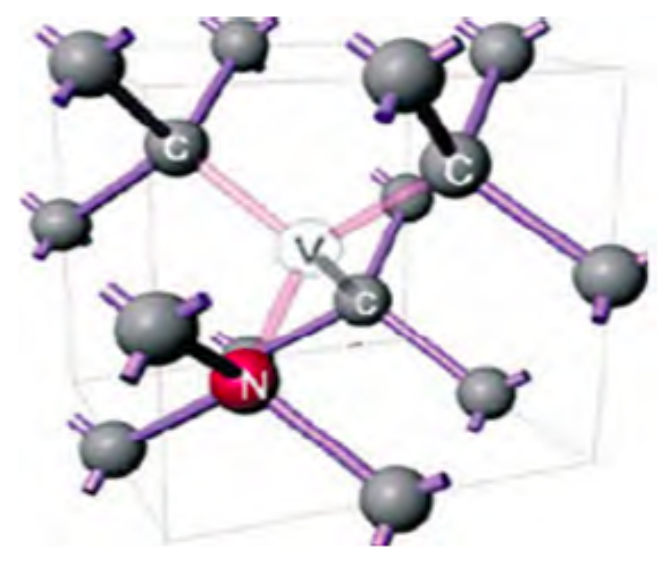

Figure 1. Structure of an NVcenter in diamond. A carbon $(\mathrm{C})$ atom is replaced by a nitrogen $(\mathrm{N})$ atom next to a vacancy $(\mathrm{V})$.

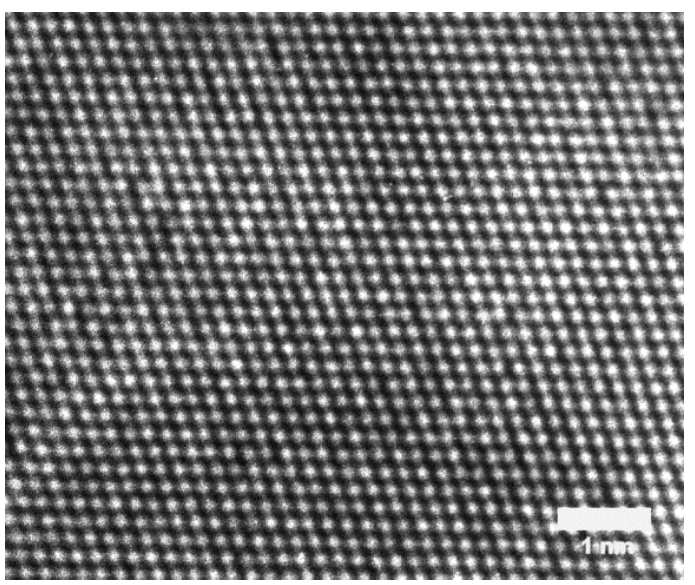

Figure 2. High resolution of diamond showing atomic contrast changes due to point defects such as vacancies in the diamond structure. 